\title{
Prevalência da Adesão Terapêutica em Pacientes Hipertensos sob Tratamento Medicamentoso em um Município do Sudoeste Baiano
}

\author{
Eudes Lima da Silva ${ }^{1}$; Nadielle Silva Bidu ${ }^{2}$; Rafael Luiz de Araújo Rodrigues ${ }^{3}$; Rodrigo Santos Damascena ${ }^{4}$
}

\begin{abstract}
Resumo: A hipertensão arterial é umas das doenças crônicas mais frequentes da atualidade e um importante fator de risco para o desencadeamento de patologias e complicações mais graves como infarto agudo do miocárdio e acidente vascular cerebral. Para evitar esses agravos, a adesão ao tratamento medicamentoso e não medicamentoso é imprescindível. Assim, este estudo buscou identificar a adesão terapêutica medicamentosa em pacientes hipertensos na Unidade Básica de Saúde, bem como os fatores diretamente relacionados ao paciente que influencia a sua não adesão. Foi realizada uma pesquisa de caráter transversal com aplicação de questionário em 150 pacientes hipertensos usuário de Unidade de Saúde da Família. A maioria dos participantes era do sexo feminino (40,7\%), pardos $(50,6 \%)$, com ensino fundamental incompleto (48,0\%) e recebiam até um salário mínimo (48,0\%). 76,7\% afirmaram não ter dificuldade de acesso à unidade de saúde e 59,3\% que há dificuldade de acesso ao medicamento. Dos pacientes estudados, $72,7 \%$ assumem fazer uso de outros medicamentos sem conhecimento médico e 70,0\% não receberam nenhuma recomendação especial sobre o tratamento de hipertensão. Observou-se que há uma baixa adesão ao tratamento medicamentoso tanto por conta da dificuldade de acesso ao tratamento quanto pela falta de entendimento sobre a própria saúde e patologia.
\end{abstract}

Palavras chave: Hipertensão arterial sistêmica; Tratamento; Adesão medicamentosa; Pacientes hipertensos;

\section{Prevalence of Therapeutic Adhesion in Hypertensive Patients under Medicinal Treatment in a Municipality of Southwest Bahia}

\begin{abstract}
Arterial hypertension is one of the chronic diseases more common in the present days and a significant risk factor to the development of pathologies and severe complications like heart attack and stroke. To avoid this grievance, the adherence to drug and no drug treatment is indispensable. So, this study aimed to identify the therapeutic drug adhesion in hypertensive patients in the Basic Health Unit, and the factors directly connected with the patient that influence the adhesion. A cross-sectional study was realized with the application of a questionnaire in 150 hypertensive patients of the Family Health Unit. Most of the participants were female (40,7\%), brown $(50,6 \%)$, with incomplete fundamental school $(48,0 \%)$ and received up to a minimum wage. $767 \%$ declared do not have difficulty accessing the health unit, and 59,3\% have challenging to access of the medicines. Of the studied patients, $72,7 \%$ assert to make use of other medicines without physician knowledge, and 70,0\% do not receive any special recommendation about the hypertense treatment. It was observed that there is low adherence to drug treatment because of the difficulty of access to treatment and lack of understanding about their health and pathology.
\end{abstract}

Keywords: Systemic arterial hypertension; Treatment; Drug adhesion; Hypertensive patients;

\footnotetext{
${ }^{1}$ Faculdade Independente do Nordeste-FAINOR, Vitória da Conquista, Bahia, Brasil. eudes_lds@hotmail.com ${ }^{2}$ Centro de Pesquisa Gonçalo Moniz, Fundação Oswaldo Cruz (FIOCRUZ), Salvador, Bahia, Brasil.

${ }^{3}$ Especialização em Pericias Criminais pelo Centro Educacional do Sudoeste da Bahia, Brasil. Coordenador da Assistência Farmacêutica do Secretaria Municipal de Saúde, Brasil

${ }^{4}$ Mestre em saúde pública pela Fiocruz. Especialista em Microbiologia Clínica. Especialista em saúde pública com ênfase em PSF. Especialista em gestão da assistência farmacêutica. Especialista em farmácia clínica e hospitalar. Graduação em ciências farmacêuticas pela Universidade Estadual de Feira de Santana.
} 


\section{Introdução}

A Hipertensão Arterial Sistêmica (HAS) é uma doença crônica controlável com alto índice de ocorrência no Brasil e no mundo (BEZERRA; LOPES; BARROS, 2014; BRASIL, 2013). Embora seja uma patologia assintomática, ela é um importante fator de risco modificável relacionado com o desenvolvimento de doenças cardiovasculares, coronarianas, cerebrovasculares, vasculares periféricas e renais (SILVA et al., 2016; BEZERRA; LOPES; BARROS, 2014; MENDES et al., 2013; LIMA; MEINERS; SOLER, 2010). Desta forma, interfere de modo deletério na qualidade de vida do paciente acometido, e, embora, haja considerável avanço em seu tratamento, o sucesso terapêutico em seu controle é limitado, sendo a HAS considerada um importante problema de saúde pública (SILVA et al., 2016; TAVARES et al., 2016; BEZERRA; LOPES; BARROS, 2014).

O tratamento para a HAS consiste em significativas mudanças no estilo de vida e no uso contínuo de medicamentos anti-hipertensivos (BEZERRA; LOPES; BARROS, 2014; BRUNE et al., 2014). Esta mudança no estilo de vida está relacionada com a adoção de hábitos adequados e saudáveis como o baixo consumo de cloreto de sódio, a prática constante de exercícios físicos, a redução do peso corporal e a diminuição na ingestão de bebidas alcoólicas (BRUNE et al., 2014). Já os medicamentos anti-hipertensivos desempenham ação terapêutica por meio de mecanismos distintos que intervêm na fisiopatologia da hipertensão, diminuindo tanto a pressão arterial dos hipertensos como, comprovadamente, a incidência de eventos cardiovasculares (BRASIL, 2018).

Conforme observado, a adesão ao plano terapêutico é um processo multidimensional relacionado com o comportamento do paciente com relação à sua saúde, com fatores socioeconômicos, com a doença, com os sistemas de saúde e, inclusive, com o tratamento medicamentoso prescrito (LIBERATO et al., 2014; LIMA; MEINERS; SOLER, 2010; OMS, 2003). A não adesão ao esquema de tratamento anti-hipertensivo é considerado como uma das principais causas de ocorrência de risco de eventos cardiovasculares e hospitalizações desnecessárias no Sistema Único de Saúde (SUS) com despesas onerosas aos cofres públicos, além da possibilidade de causar a morte do paciente hipertenso (SOUZA et al., 2018).

Por conta desses múltiplos fatores relacionados, a taxa de abandono ao tratamento é crescente com o tempo, sendo, portanto, imprescindível que se consiga a adesão do paciente à terapia para que o sucesso ao tratamento anti-hipertensivo seja garantido e, assim, a prevenção na ocorrência dos agravos e complicações das doenças relacionadas com a HAS não controlada 
(LIMA; MEINERS; SOLER, 2010; CASTRO et al., 2006). Alguns fatores determinantes para a não aderência ao tratamento farmacológico são a ausência de conhecimento sobre a doença, a polifarmácia, os efeitos adversos e as incontáveis doses diárias, sendo a identificação desses fatores de suma importância para o emprego das estratégias de reversão desse quadro (SOUZA et al., 2018; FAVA et al., 2014).

Há muitas evidências de que o aumento da frequência de utilização dos medicamentos gera um impacto negativo sobre a adesão à terapêutica, o que pode agravar o estado de saúde desses pacientes e, como consequência, haver a adição de outros medicamentos ao já complicado esquema terapêutico. Assim, com o intuito de evitar esse quadro e aumentar a adesão dos pacientes hipertensos à terapêutica, os profissionais da saúde em serviço nas unidades de saúde podem adicionar ao processo de atendimento dessa população uma avaliação sistemática da adesão ao tratamento e, assim, promover a adesão e melhorar a qualidade de vida destes (REMENDI; CABRERA; SOUZA, 2014).

\section{Metodologia}

\section{Tipo de estudo}

Pesquisa de caráter transversal, com abordagem quantitativa do tipo descritiva e exploratória, aprovada pelo Comitê de Ética e Pesquisa da Faculdade Independente do Nordeste em 21 de agosto de 2018 sob parecer de número 2.833.739. Todos os pacientes do estudo assinaram o termo de consentimento referente à pesquisa.

\section{Participantes}

Foram realizadas entrevistas estruturadas com 150 pacientes da Unidade de Saúde da Família (USF) situada em um município no sudoeste baiano. Os critérios de inclusão foram: ser hipertenso, ter idade entre 20 e 79 anos, estar agendando para consultas na unidade de saúde e fazer uso de medicamento anti-hipertensivo. Não sendo incluídos qualquer paciente que não atendia aos critérios de inclusão definidos previamente. 


\section{Instrumento e procedimentos}

Foi aplicado um questionário elaborado conforme as necessidades do estudo pelos autores da pesquisa contendo questões relacionadas a se o paciente tem dificuldade de acesso à unidade ou a medicamentos na unidade de saúde; se faz uso de medicamentos não prescritos pelo médico; e se esquece de tomar ou se suspendeu o uso do medicamento sem informar o médico.

\section{Análise estatística}

Os dados foram digitalizados e tratados utilizando-se o programa Microsoft ${ }^{\circledR}$ Excel $^{\circledR}$ 2018, sendo considerada a distribuição percentual das respostas dados às questões presentes no instrumento de pesquisa utilizado.

\section{Resultados e Discussão}

Os dados demográficos dos pacientes participantes da pesquisa são mostrados na tabela 1, a seguir:

Tabela 1. Dados sociodemográficos dos pacientes hipertensos submetidos ao estudo.

\begin{tabular}{llcc}
\hline Variáveis & & F & $\%$ \\
\hline \multirow{2}{*}{ Sexo } & Masculino & 61 & 40,7 \\
& Feminino & 89 & 59,3 \\
\multirow{3}{*}{ Estado civil } & Solteiro & 72 & 48,0 \\
& Casado & 31 & 20,7 \\
& Viúvo & 24 & 16,0 \\
Raça & Divorciado & 23 & 15,3 \\
(auto declarado) & Parda & 76 & 50,6 \\
& Branca & 49 & 32,7 \\
Trabalho & Negra & 25 & 16,7 \\
& Sim & 82 & 54,6 \\
Renda familiar & Não & 61 & 40,7 \\
& Aposentado & 07 & 4,7 \\
& Até 1 salário mínimo & 72 & 48,0 \\
& De 1 a 3 salários mínimo & 57 & 38,0
\end{tabular}




\begin{tabular}{lllc} 
& De 3 a 6 salários mínimo & 06 & 4,0 \\
Nenhum & 15 & 10,0 \\
& Ensino fundamental completo & 28 & 18,6 \\
& Ensino fundamental incompleto & 72 & 48,0 \\
Ensino médio completo & 21 & 14,0 \\
Escolaridade & Ensino médio incompleto & 09 & 6,0 \\
& Ensino superior completo & 03 & 2,0 \\
& Sem escolaridade & 16 & 10,7 \\
\hline
\end{tabular}

F: frequência absoluta. Fonte: dados da pesquisa

Conforme observado, há predominância de pacientes do sexo feminino, 89 (59,6\%); com renda familiar de até um salário mínimo, 72 (48,0\%); e com ensino fundamental incompleto, $72(48,0 \%)$.

Vários estudos também encontraram essa predominância do sexo feminino (SOUZA et al., 2018; ALMEIDA et al., 2017; ROCHA; BORGES; MARTINS, 2017; FERREIRA; GRAÇA; CALVINHO, 2016; TAVARES et al., 2016; BRUNE; FERREIRA; FERRATI, 2014; MOTTA et al., 2014; REMENDI; CABRERA; SOUZA, 2014), a qual, provavelmente, pode ser explicada por vários fatores como: o fato delas serem mais preocupadas com sua própria saúde do que os homens; possuírem uma maior percepção aos cuidados com a saúde, por isso, são maioria entre os usuários dos serviços de saúde; e a dupla jornada de trabalho (profissional e doméstico) intimamente relacionada com estresse, que é um fator de risco para a alteração da pressão arterial.

Segundo Esperandio e col. (2013) há uma maior tendência de hipertensos entre os pacientes solteiros por conta da interferência no bem estar emocional e no estilo de vida causados pela instabilidade conjugal e o estresse relacionado, e que, em indivíduos casados, pode haver incentivo do cônjuge à prática de comportamentos saudáveis. Nosso estudo também encontrou uma maior frequência de pacientes hipertensos solteiros, 72 (48\%), mas como este estudo só teve a participação de pacientes hipertensos, não há como avaliar se o estado civil é fator associado com o desenvolvimento da HAS.

Com relação à raça, a maioria dos pacientes se autodeclarou pardo, $76(50,6)$, o que corrobora com os achados de Almeida e colaboradores (2017) e Rocha; Borges; Martins (2017), e confirmados pela VI Diretrizes Brasileira de Hipertensão (2010) que assinala serem os indivíduos da cor não branca duas vezes mais propícios de terem HAS. 
A maioria da população do estudo pertencia à classe socioeconômica média baixa, que é caracterizada por uma população com poucos recursos econômicos, no caso deste estudo recebem até um salário mínimo, 72 (48,0\%); baixa escolaridade, no estudo maioria tem ensino fundamental incompleto, $72(48,0 \%)$; e baixo estatuto social. Achados semelhantes aos de Ferreira; Graça; Calvinho (2016) e aos de Rocha; Borges; Martins (2017), os quais descrevem que a baixa escolaridade pode comprometer o tratamento devido às dificuldades de discernimento e acato das orientações informadas pela equipe de saúde, estando esses indivíduos mais expostos aos fatores de risco de ocorrência e agravo da HAS. Além disso, o poder aquisitivo baixo pode influenciar negativamente no acesso ao medicamento quando este não é disponibilizado na unidade de saúde.

A tabela 2 mostra os dados referentes às dificuldades de acesso ao atendimento e ao medicamento na unidade de saúde.

Tabela 2. Frequência de consultas e dificuldades de acesso à unidade de saúde e aos medicamentos relatados no estudo.

\begin{tabular}{llll}
\hline Variáveis & & F & $\%$ \\
\hline \multirow{2}{*}{ Última consulta } & Menos de 3 meses & 58 & 38,7 \\
& De 4 a 6 meses & 50 & 33,3 \\
Dificuldade de acesso a unidade de saúde? & Mais de 1 ano & 42 & 28,0 \\
& Sim & 35 & 23,3 \\
Dificuldade de acesso a medicamentos na Unidade? & Não & 115 & 76,7 \\
& Sim & 89 & 59,3 \\
\hline
\end{tabular}

F: frequência absoluta. Fonte: dados da pesquisa

Pela HAS ser uma doença crônica, o paciente hipertenso deve ser avaliado periodicamente para que o profissional prescritor e os outros profissionais de saúde envolvidos no tratamento possam identificar possíveis efeitos colaterais, interações medicamentosas e necessidade de adequação da dose terapêutica. Segundo o parecer do Conselho Federal de Medicina (CFM n ${ }^{\circ}$ 12/06) "pacientes crônicos em uso de medicamentos de uso contínuo devem ser avaliados por seus médicos, no máximo, a cada 90 (noventa) dias, em vista da boa prática médica e das adequações necessárias" (CFM, 2006). Assim, apenas a minoria dos pacientes do estudo, $58(38,7 \%)$, estavam adequados com a frequência de consultas regular para verificação das condições de saúde. 
Um dos motivos para a maioria dos pacientes, 92 (61,3\%), estarem apenas realizando as consultas após os 3 meses pode ser a dificuldade de acesso à unidade de saúde. Fava e col. (2014) afirmam que a dificuldade de acesso ao sistema público é um dos fatores fortemente relacionados à não adesão ao tratamento, entretanto, isso não foi observado nos dados, pois a maioria relata não encontrar dificuldades de acesso à unidade, 115 (76,7\%). Portanto, outros motivos podem estar relacionados à demora de realização das consultas na unidade como o tempo, pois a maioria dos pacientes relatam trabalhar, $82(54,6 \%)$, e a falta de entendimento do que seria a dificuldade de acesso.

Um outro fator importantíssimo para o seguimento do tratamento prescrito e indicador da qualidade e resolutividade do sistema de saúde é o acesso aos medicamentos (FAVA et al., 2014). Mais da metade dos pacientes estudados, 89 (59,3\%), indicaram que há dificuldade de acesso aos medicamentos da unidade. Sem o acesso ao medicamento torna-se difícil a adesão ao tratamento e consequente controle da pressão arterial. Uma solução seria a aquisição do medicamento fora da unidade, mas, conforme observado anteriormente, o baixo poder aquisitivo da maioria dos pacientes inviabiliza essa possibilidade.

Conforme dados demonstrados na tabela 3, dos 150 pacientes estudos, 109 (72,7\%) fizeram uso de algum outro medicamento sem prescrição médica. Devido à característica de polifarmácia da hipertensão arterial, o uso de outros medicamentos sem conhecimento do médico pode acarretar no aparecimento de outros agravos à saúde desse paciente. Somado a isso, $105(70,0 \%)$ pacientes afirmaram não terem recebido do médico nenhuma recomendação especial de cuidados. Segundo Tavares e col. (2016), a educação em saúde é uma ferramenta que pode ser muito útil para reduzir ou evitar o impacto que os fatores relacionados com a adesão ou não ao tratamento medicamentoso da HAS. Assim, a informação médica juntamente com o acompanhamento e monitorização da saúde do paciente hipertenso, por meio da atenção farmacêutica realizada pelo farmacêutico, contribui enormemente para a identificação das dificuldades relacionadas à adesão e informa o paciente sobre sua patologia e seu cuidado.

Tabela 3. Distribuição da amostra segundo fatores relacionados à adesão terapêutica medicamentosa.

\begin{tabular}{llcc}
\hline Variáveis & & F & \% \\
\hline O Sr. já usou algum medicamento sem que o médico tenha lhe & Sim & 109 & 72,7 \\
receitado? & Não & 38 & 25,3 \\
& Não respondeu & 03 & 2,0
\end{tabular}




\begin{tabular}{lllc}
\hline Você esquece de tomar seus Remédios ? & Sim & 55 & 36,7 \\
& Não & 94 & 62,6 \\
& Não respondeu & 01 & 0,7 \\
Você já parou de tomar seus medicamentos ou diminuiu a dose sem & Sim & 59 & 39,3 \\
avisar seu médico porque se sentia pior quando os tomava? & Não & 88 & 58,7 \\
& Não respondeu & 03 & 2,0 \\
Quando viaja ou sai de casa, as vezes esquece de levar seus & Sim & 75 & 50,0 \\
medicamentos? & Não & 74 & 49,3 \\
Quando seu médico lhe passou um destes medicamentos, ele fez & Não respondeu & 01 & 0,7 \\
alguma recomendação especial de cuidados durante o uso? & Sim & 45 & 30,0 \\
\hline F: frequência absoluta. Fonte: dados da pesquisa & Não & 105 & 70,0 \\
\hline
\end{tabular}

F: frequência absoluta. Fonte: dados da pesquisa

Como a maioria dos pacientes estudados não esquecem de tomar os medicamentos prescritos, 94 (62,6\%), o que provavelmente desencadeia a não adesão medicamentosa nesses pacientes é o aparecimento de algum efeito adverso que faz com que os hipertensos reduzam ou deixem de tomar o medicamento, 59 (39,3\%). Como supracitado, a desinformação do paciente sobre a sua condição e a importância dos medicamentos é um agravante no quadro hipertensivo, pois faz com que o paciente tome essa decisão de interromper o tratamento medicamentoso. Desta forma, há uma piora na evolução clínica e na qualidade de vida desses pacientes, o que desencadeia um aumento nos gastos e nos índices de morbimortalidade dessa patologia (MOTTA et al., 2014).

\section{Conclusão}

O manejo da não adesão ao tratamento é cada vez mais necessário no cotidiano dos serviços de saúde, pois a utilização correta dos medicamentos é fundamental para a redução de grande parte das doenças crônicas não transmissíveis (DCNT) como a hipertensão. Observouse, no estudo, um insuficiente grau de adesão ao tratamento para a hipertensão arterial, o que pode desencadear o surgimento de diversas complicações. Desta forma, é necessário o planejamento e a implementação de sistemas de educação junto com os pacientes, tendo o objetivo de esclarecer a condição de saúde de cada paciente bem como a importância do tratamento realizado de forma adequada, além de permitir uma conscientização pelos 
profissionais de saúde na promoção de autonomia, aceitação, conhecimento e adaptação do paciente em relação a sua própria doença.

\section{Referências}

ALMEIDA, A. S. de et al. Lifestyle and socioeconomic profile of hypertensive patients. Journal of Nursing, v. 11, n. 12, p. 4826-4837, 2017.

BEZERRA, A. S. de M.; LOPES, J. de L.; BARROS, A. L. B. L. de. Adesão de pacientes hipertensos ao tratamento medicamentoso. Revista Brasileira de Enfermagem, v. 67, n. 4, p. $550-555,2014$.

BRASIL. Linha Guia de Hipertensão Arterial. Secretaria de Estado de Saúde do Paraná, Superintendência de Atenção à Saúde, 2 ed., Curitiba: SESA, 2018.

BRASIL, Ministério da Saúde. Pesquisa Nacional de Saúde 2013 Percepção do estado de saúde, estilos de vida e doenças crônicas. Brasília, DF, 2013.

BRUNE, M. F. S. S., FERREIRA, E. E., FERRARI, C. K. B. O Método Dáder na atenção farmacêutica em pacientes hipertensos no município de Pontal do Araguaia-MT, Brasil. O Mundo da Saúde, v. 38, n. 4, p. 402-409, 2014.

CASTRO, M. S. de et al. Contribuição da atenção farmacêutica no tratamento de pacientes hipertensos. Revista Bras. Hipertensão, v. 13, n. 3, p. 198-202, 2006.

CFM. Parecer $n^{\circ}$ 12/06. Orientação aos médicos quanto à prescrição de medicamentos de uso contínuo. 2006. Disponível em: https://sistemas.cfm.org.br/normas/arquivos/pareceres/BR/2006/12_2006.pdf

ESPERANDIO, E. M. et al. Prevalência e fatores associados à hipertensão arterial em idosos de municípios da Amazônia Legal, MT. Revista Brasileira de Geriatria e Gerontologia, v. 16, n. 3, p. 481-493, 2013.

FAVA, S. M. C. L. et al. Fatores relacionados à adesão ao tratamento da hipertensão arterial sistêmica. Revista Rene, v. 15, n. 2, p. 354-361, 2014.

FERREIRA, R. S. da S.; GRAÇA, L. C. C. da; CALVINHO, M. de L. S. E. Adesão ao Regime Terapêutico de Pessoas com Hipertensão Arterial em Cuidados de Saúde Primários. Revista de Enfermagem Referência, v. Série IV, n. 8, p. 7-15, 2016.

LIBERATO, S. et al. Relação entre adesão ao tratamento e qualidade de vida: revisão integrativa da literatura. Revista Eletrônica de Enfermagem, v. 16, n. 1, p. 191-8, 2014.

LIMA, T. DE M.; MEINERS, M. M. M. DE A.; SOLER, O. Perfil de adesão ao tratamento de pacientes hipertensos atendidos na Unidade Municipal de Saúde de Fátima, em Belém, Pará, Amazônia, Brasil. Revista Pan-Amazônica de Saúde, v. 2, n. 1, p. 113-120, 2010. 
MENDES, L. M. E O. et al. Fatores associados à não adesão ao tratamento da hipertensção arterial sistêmica: uma revisão integrativa. Revista Univap, v. 20, n. 35, p. 56-68, 2014.

MOTTA, P. G. da et al. Adesão medicamentosa ao tratamento da hipertensão de pacientes do hiperdia em Ipatinga e Timóteo, Minas Gerais. Revista UNINGÁ, v. 40, n. 1, p. 91-103, 2014.

OMS, Organização Mundial de Saúde. Cuidados inovadores para condições crônicas: componentes estruturais de ação: relatório mundial. Brasília (DF): 2003. Disponível em: http://www.who.int/diabetesactiononline/about/icccportuguese.pdf

REMONDI, F. A.; CABRERA, M. A. S.; SOUZA, R. K. T. DE. Não adesão ao tratamento medicamentoso contínuo: prevalência e determinantes em adultos de 40 anos e mais. Cadernos de Saúde Pública, v. 30, n. 1, p. 126-36, 2014.

ROCHA, M. L. F.; BORGES, J. W.; MARTINS, M. S. Adesão ao tratamento da hipertensão arterial entre usuparios da estratégia de saúde da família em um município do Piauí. APS, v. 20, n. 1, p. 6-20, 2017.

SILVA, A. P. da et al. Fatores associados à não adesão ao tratamento da hipertensão arterial sistêmica: uma revisão integrativa. Revista de Pesquisa: Cuidado é Fundamental Online, v. 8, n. 1, p. 4047-4055, 2016.

SOUSA, R. de C. et al. Particularities of hypertensive elderly people to medicinal treatment adherence. Journal of Nursing, v. 12, n. 1, p. 216-23, 2018.

TAVARES, D. M. dos S. et al. Qualidade de vida e adesão ao tratamento farmacológico entre idosos hipertensos. Revista Brasileira de Enfermagem, v. 69, n. 1, p. 134-141, 2016.

VI Diretrizes Brasileiras de Hipertensão. Arq. Bras. Cardiol., São Paulo, v. 95, n. 1, supl. 1, p. I-III, 2010 .

\section{Como citar este artigo (Formato ABNT):}

SILVA, Eudes Lima da; BIDU, Nadielle Silva; RODRIGUES, Rafael Luiz de Araújo; DAMASCENA, rodrigo Santos. Prevalência da Adesão Terapêutica em Pacientes Hipertensos sob Tratamento Medicamentoso em um Município do Sudoeste Baiano. Id on Line Rev.Mult. Psic., 2018, vol.12, n.42, Supl. 1, p. 749-759. ISSN: 1981-1179.

Recebido: $14 / 11 / 2018$

Aceito: $17 / 11 / 2018$ 